University of Wollongong

Research Online

Faculty of Social Sciences - Papers (Archive) Faculty of Arts, Social Sciences \& Humanities

2013

Short-term training in the Go/Nogo task: Behavioural and neural changes depend on task demands

Nicholas Benikos

University of Wollongong, npb95@uowmail.edu.au

Stuart J. Johnstone

University of Wollongong, sjohnsto@uow.edu.au

Steven J. Roodenrys

University of Wollongong, steven@uow.edu.au

Follow this and additional works at: https://ro.uow.edu.au/sspapers

Part of the Education Commons, and the Social and Behavioral Sciences Commons

Research Online is the open access institutional repository for the University of Wollongong. For further information contact the UOW Library: research-pubs@uow.edu.au 


\title{
Short-term training in the Go/Nogo task: Behavioural and neural changes depend on task demands
}

\begin{abstract}
Neural activity underlying executive functions is subject to modulation as a result of increasing cognitive demands and practice. In the present study, we examined these modulatory effects by varying task difficulty, as manipulated by reaction time deadline (RTD), on inhibitory control during a single Go/Nogo training session (8 blocks; $70 \% \mathrm{Go}$ ). Sixty adults were randomly assigned to one of three task difficulty conditions: High $(n=20)$, Medium $(n=20)$ and Low $(n=20)$, with RTDs of 300, 500 or 1000 ms, respectively. Task performance, Event-related potentials (ERPs) and task-related arousal (indexed by skin conductance level) were examined for training effects. Results indicated that improvements in behavioural Go/Nogo proficiency were optimised during conditions of moderate rather than low or high inhibitory demands. An across-session increase in task-related arousal did not differ between conditions, indicating a generalised increase in the mobilisation of mental resources with time-on-task. In contrast, training-related changes in ERPs were dependent on task demands such that the Low task difficulty condition showed an enhanced centroparietal Nogo P2, while a training-induced augmentation in the Nogo > Go P3 effect was greater in the High than Medium condition. The High condition also showed the greatest reduction in the Nogo N1. Although further research is needed in this area, these findings implicate the potential key role of task difficulty in training inhibitory control and suggest that practicerelated changes are reflected by qualitative changes in brain activity.
\end{abstract}

\section{Keywords}

nogo, go, training, demands, depend, changes, term, neural, short, behavioural, task

\section{Disciplines}

Education | Social and Behavioral Sciences

\section{Publication Details}

Benikos, N., Johnstone, S. J. \& Roodenrys, S. J. (2013). Short-term training in the Go/Nogo task:

Behavioural and neural changes depend on task demands. International Journal of Psychophysiology, 87 (3), 301-312. 


\title{
Short-term training in the Go/Nogo Task: Behavioural and Neural Changes Depend on Task Demands
}

\author{
Nicholas Benikos \\ University of Wollongong, npb95@uow.edu.au
}

Stuart J. Johnstone

University of Wollongong, stuart_johnstone@uow.edu.au

Steven J. Roodenrys

University of Wollongong, steven@uow.edu.au

Publication details: Benikos, N., Johnstone, S. J. \& Roodenrys, S. J. (2013). Short-term training in the Go/Nogo Task: Behavioural and Neural Changes Depend on Task Demands. International Journal of Psychophysiology, 87 (3), 301-310. 


\begin{abstract}
Neural activity underlying executive functions is subject to modulation as a result of increasing cognitive demands and practice. In the present study, we examined these modulatory effects by varying task difficulty, as manipulated by reaction time deadline (RTD), on inhibitory control during a single Go/Nogo training session ( 8 blocks; $70 \% \mathrm{Go}$ ). Sixty adults were randomly assigned to one of three task difficulty conditions: High $(n=20)$, Medium $(n=20)$ and Low (n = 20), with RTDs of 300, 500 or 1000 ms, respectively. Task performance, Event-related potentials (ERPs) and task-related arousal (indexed by Skin Conductance Level) were examined for training effects. Results indicated that improvements in behavioural Go/Nogo proficiency were optimised during conditions of moderate rather than low or high inhibitory demands. An across-session increase in task-related arousal did not differ between conditions, indicating a generalised increase in the mobilisation of mental resources with time-on-task. In contrast, training-related changes in ERPs were dependent on task demands such that the Low task difficulty condition showed an enhanced centroparietal Nogo P2, while a training-induced augmentation in the Nogo > Go P3 effect was greater in the High than Medium condition. The High condition also showed the greatest reduction in the Nogo N1. Although further research is needed in this area, these findings implicate the potentially key role of task difficulty in training inhibitory control and suggest that practice-related changes are reflected by qualitative changes in brain activity.
\end{abstract}

\title{
Keywords:
}

Training; Practice; Task difficulty; ERPs; Inhibition; N2; P3; Arousal 


\section{Introduction}

Everyday functioning requires the ability to deliberately inhibit dominant, automatic, or prepotent responses (Dagenbach and Carr, 1994; Dempster and Corkill, 1999). Localised to fronto-striatal networks (Aron et al., 2007; Aron et al., 2004), inhibitory control is crucial for the proper performance of many other higher-order cognitive functions, including working memory (Hester et al., 2004), task switching (Monsell, 2003) and action monitoring (Cooper and Shallice, 2000). Unfortunately, this capacity is susceptible to impairment with deficits linked to diverse spheres of atypical functioning, from excess consumption of food (Blumenthal and Gold, 2012) and alcohol (Wiers et al., 2007), to several psychiatric and neurological disorders, such as Attention-deficit/Hyperactivity disorder (ADHD; Smith et al., 2004) and Huntington's disease (Beste et al., 2008).

Intensive research over the last two decades has revealed that the neural mechanisms which underlie executive functions are amenable to training and experience (for a review see Kelly and Garavan, 2005), superseding the traditionally held belief that the human adult brain is hardwired and resistant to change (Buonomano and Merzenich, 1998; Kelly et al., 2006b; Raskin et al., 2011). Consequently, fundamental research investigating the training-induced alterations in these abilities may not only determine the extent to which neuroplastic changes are available to healthy adults (Kelly et al., 2006a; Kelly and Garavan, 2005), but also aid in remediating atypical neural processes (Kelly et al., 2006a; Kujal and Näätänen, 2010). However, despite a recent upsurge of positive findings regarding the training of other executive functions (e.g. working memory, attention, task switching; for reviews see Cramer et al., 2011; Green and Bavelier, 2008; Kujal and Näätänen, 2010), the literature investigating the training of inhibitory control has been mixed.

For example, some studies report direct training-related improvements in inhibitory performance (Dowsett and Livesey, 2000; Schapkin et al., 2007; Thorell et al., 2009; Verbruggen and Logan, 2008), while others have found more indirect effects, such as a reduction in the consumption of food and alcohol after participants were trained to inhibit food and alcohol cues embedded in Go/Nogo and Stop-Signal tasks (Houben, 2011; Houben and Jansen, 2011; Houben et al., 2011; Veling et al., 2011). Despite this, several other investigations have reported no significant change (Jodo and Inoue, 1990; Kelly et al., 2006b; Rueda et al., 2005; Tomporowski, 2003), or even declines in performance with practice (Kato et al., 2009; Manuel et al., 2010), and no transfer to untrained tasks (Manuel et al., 2010; Thorell et al., 2009). In sum, despite some studies showing promising results, the question of how to reliably attain training-induced improvements in inhibitory control is still not clear - highlighting the 
fragmentary nature of our current understanding, and pointing to the need for further work in this area.

In a complementary line of research, a number of previous working memory (WM) training studies have consistently demonstrated improvements in cognition (Chein and Morrison, 2010; McNab et al., 2009; Olesen et al., 2004) and overt behaviour post-training (Klingberg et al., 2005; Klingberg et al., 2002). Notably, these studies highlighted that a key ingredient of productive training was the enhancement of task difficulty (Klingberg, 2010; Lindqvist and Thorell, 2009; Thorell et al., 2009), while the training of WM at low levels of capacity does not lead to a substantial improvement (for a combined WM and inhibition training study see Johnstone et al., 2010; Klingberg et al., 2005; Klingberg et al., 2002). Similarly, variations in task difficulty also appear to be important for the training of other cognitive abilities (e.g. attention, perception; Kelley and Yantis, 2009; Wang et al., 2010). While we have previously demonstrated that Go/Nogo task difficulty can be successfully manipulated via reaction time deadline (RTD; Benikos et al., in press), whether different variations in RTD can augment the training of prepotent response inhibition has yet to be systematically investigated. Previous research has either not manipulated task difficulty (e.g. Jodo and Inoue, 1990), or have used auto-adaptive difficulty manipulations (Manuel et al., 2010), leaving the question as to the optimal difficulty level required for learning in the Go/Nogo task open. Thus, the primary aim of the present study was to investigate the effect of varying degrees of task difficulty (i.e. Low, Medium and High) during the training of the Go/Nogo task, rather than simply assuming optimal learning at a single level.

Although variations in task performance offer a global measure of training-related changes in inhibitory control, they do little to provide an insight into the underlying neural mechanisms. In contrast, Event-related potentials (ERPs) allow a detailed examination of these processes, with ERP amplitude and latency sensitive to neuroplastic changes in brain activity (Kujal and Näätänen, 2010; Lillard and Erisar, 2011). Among the most commonly investigated ERPs in the Go/Nogo task is the Nogo N2, a frontally maximal negative component peaking around $200 \mathrm{~ms}$ after the onset of inhibition-evoking stimuli (Johnstone et al., 2005; Smith, 2011), and the Nogo $\mathrm{P} 3$, a positive component that has a more anterior topography than the Go P3, and peaks approximately 300 ms post-stimulus (Randall and Smith, 2011; Smith and Douglas, 2011). The link between the N2 and P3 to inhibitory processing is the subject of debate (Bruin et al., 2001; Nieuwenhuis et al., 2003). Some argue that the inhibition process is best reflected by the N2 (Falkenstein et al., 1999; Kok, 1986), while others suggest instead that the P3 is the more likely candidate (Randall and Smith, 2011; Smith, 2011; Smith and Douglas, 2011; Smith et al., 2006, 2007, 2008; Smith et al., 2010). 
The literature investigating the effect of inhibition training on neural activity has been varied in regard to methodologies and outcomes. Jodo and Inoue (1990) found that Go reaction time and Nogo P3 latency were significantly shortened after six days of practice (200 trials per day) - in line with previous theoretical viewpoints suggesting more efficient processing with training (Neubauer and Fink, 2009). However, given that no results for task performance were reported, a link between more efficient inhibitory performance and the Nogo P3 is unable to be made. Schapkin and colleagues (2007) found a reduction in Nogo errors, with a corresponding increase in the Nogo N2 after the first three daily training sessions ( 200 trials per day) of a three week training protocol, which they interpreted as a practice-related strengthening of the inhibition mechanism (for a similar finding using fMRI see Kelly et al., 2006b). No further change was reported by the conclusion of the training. Finally, more efficient early low-level processing has been suggested by Manuel and colleagues (2010), who found a reduction in left parietal activity to Nogo stimuli at 61-104 ms following thirty minutes of Go/Nogo task practice (528 trials total).

In sum, the literature does not appear to paint a straightforward picture of the neural changes that should be expected as a result of training inhibitory control. Furthermore, the above mentioned studies had a variety of shortcomings. First, it has been suggested that response inhibition may not be solely manifested by variations in the N2 and P3, but that earlier components in the waveform such as the N1 and P2 play a crucial role in determining inhibition success (Roche et al., 2005; Thomas et al., 2009). Yet these components have not been investigated in the context of inhibition training. Secondly, previous work has generally only employed Pre/Post designs (Jodo and Inoue, 1990; Kelly et al., 2006a), which do little in the way of understanding the time-course of training effects. For optimal paradigm design it would be advantageous to isolate the time required to elicit positive training effects (Cramer et al., 2011). Finally, most studies have typically only included eleven or fewer participants (Jodo and Inoue, 1990; Manuel et al., 2010; Schapkin et al., 2007), making the generalisability of their findings and brain-behaviour correlations difficult to assess.

\subsection{The Present Study}

The primary aim of the present study was to examine the effect of varying task difficulty, as manipulated by RTD, on the training of inhibitory control using task performance and inhibition-related ERPs. Participants were divided into one of three Go/Nogo task difficulty conditions: Low (1000 ms), Medium (500 ms) and High (300 ms). Since previous Go/Nogo studies have demonstrated ceiling effects in task performance using Low difficulty RTDs (e.g. 
Johnstone et al., 2005; Smith et al., 2006), and that High task difficulty generally results in performance declines (e.g. Benikos et al., in press), it was hypothesised that training outcomes would be optimised for the Medium task difficulty condition, with concurrent enhancements in the Nogo N2 and P3. A further focus of this study was on the potential contribution of early sensory processing to the training of inhibitory control, as indicated by the N1 and P2. While no specific predictions were made for these components, any differences found would be explored. Finally, a criticism of training paradigms manipulating task difficulty is the lack of consideration of state factors, such as task-related effort and arousal (Cramer et al., 2011; Green and Bavelier, 2008; Slagter et al., 2011). Given that these variables may be critical modulators of behaviour and task performance (e.g. Slagter et al., 2011; Tang and Posner, 2009; Yerkes and Dodson, 1908), participants provided perceived effort ratings and we recorded skin conductance level (SCL) - a well-established measure of central nervous system arousal (Barry and Sokolov, 1993).

\section{Method}

\subsection{Participants}

A total of 69 adults enrolled in the present study to fulfil an undergraduate course requirement, with three being excluded according to the selection criteria. To be included in the study, participants were required to refrain from caffeine for 2 hours prior to testing and have not taken any psychotropic substances (prescription or illegal) for 24 hours prior to testing, or no more than once a month in the previous six months. Participants were also screened for neurological disorders and all reported normal or corrected-to-normal vision.

The remaining 66 participants were randomly assigned to one of three conditions: Low, Medium or High task difficulty. Of these, data from 4 subjects were rejected either due to excessive eye artefact (3 participants, leaving an insufficient number of correct and artefact free trials available for averaging) or to faulty recording equipment (1 participant). A further two people were excluded, with one being unable to complete the testing session due to an unrelated emergency, and another for failing to perform the task properly by adopting a strategy of disregarding accuracy on Nogo trials in order to respond within the RTD. Therefore, 20 participants each were included in the final analyses for the Low (17 females, 3 males, mean age 21.23, SD 4.12), Medium (14 females, 6 males, mean age 21.5, SD 5.89) and High condition (14 females, 6 males, mean age 21.4, SD 3.32). There were no differences in age $(F(2,57)=$ $0.13, p=.877)$ or gender $\left(X^{2}(2)=0.53, p=.766\right)$ between conditions. All but 5 of the 60 
participants were right-handed. The research protocol was approved by the joint University of Wollongong and Illawarra Area Health Service Human Research Ethics Committee.

\subsection{Task}

Stimuli were delivered using Presentation (Version 11.0; Neurobehavioral Systems, Albany, CA, USA). Each trial began with a central fixation cross (+) presented for a variable interval of $500-1000 \mathrm{~ms}(\mathrm{M}=750 \mathrm{~ms})$, followed by the Go/Nogo stimulus presented in the centre of the screen for $200 \mathrm{~ms}$. A blank screen then replaced the stimulus for a variable blank period of $1250-1750 \mathrm{~ms}(\mathrm{M}=1500 \mathrm{~ms})$. Within this period, participants in the High, Medium and Low task difficulty conditions were required to respond by a button press with their right hand (irrespective of handedness) to Go stimuli within 300,500 or $1000 \mathrm{~ms}$, respectively, or to refrain from responding to Nogo stimuli. Performance feedback was provided via the subsequent fixation cross: correct responses were followed by a white fixation cross, while a red fixation cross was displayed after incorrect responses (i.e. presses to Nogo stimuli during the variable blank period, omissions and responses outside the RTD). Only presses to the Go stimulus within the predefined response window were regarded as correct.

Participants first completed an initial practice block of 30 trials (50\% Nogo). In line with previous behavioural studies demonstrating improvements in inhibitory control using a single training session (Kelly et al., 2006b; Verbruggen and Logan, 2008), participants completed eight experimental blocks ( $30 \%$ Nogo) of 100 trials each. In order to minimise habituation of the visual ERP response (particularly the P3 component, e.g. see Ravden and Polich, 1998), the selection of shapes used to represent Go and Nogo stimuli were selected from a pool of eight 2D shapes (i.e. triangle, cross, hexagon, diamond, ellipse, rectangle, star or circle). Go/Nogo shape selection was changed from block to block. Presentation of shape stimuli were counterbalanced using a Latin square design (Bradley, 1958), and Go/Nogo response assignment counterbalanced across subjects. Stimuli measured approximately $3 \times 3 \mathrm{~cm}$ and were presented on a 15" computer monitor, with participants' seated one metre away. Each block lasted approximately 3.5 minutes. In order to equate training session length between conditions, the rest period between blocks was set at 1.5 minutes for all participants. Total session time including the practice and training blocks was approximately 43 minutes. 


\subsection{Procedure}

Participants were given an outline of the testing procedure and familiarised with the laboratory equipment before informed consent was given. The experimenter emphasised that participants could withdraw at any time without penalty. They were then asked to complete a short screening questionnaire to assess vision problems, medication/psychotropic substance use, and neurological disorders. Subjects were then fitted with EEG and skin conductance recording equipment, and seated in a dimly-lit sound-attenuated and electrically-shielded testing booth An incandescent light in the booth was dimmed for the duration of the training session. An initial 3 min baseline recording was conducted while participants were asked to sit quietly with eyes closed. Subjects were then presented with a modified Go/Nogo task and were instructed that they would see one of two shapes, one representing the Go stimulus, and the other representing the Nogo stimulus. They were asked to press the button before the pre-determined RTD with the thumb of their right hand (irrespective of handedness) to Go stimuli, and to refrain from responding to Nogo stimuli. Participants were asked to "do their best" to avoid the incorrect feedback, and were encouraged to keep as still as possible and to minimise eye movements during the testing blocks. Go/Nogo shape assignment was shown on the screen and verbally confirmed by the participant prior to each block. At the end of each block, mean Go RT, the percentage of Go and Nogo errors were displayed for subjects to review. They were then asked to rate their perceived level of effort irrespective of their task performance with the question "How much effort did you use to complete that block?" and responded by a 5-point Likert scale ranging from: 1 = Very little, $2=$ Moderate effort, and $5=$ Everything I had ${ }^{1}$. Prior to the first rating a basic example was shown to the subject to ensure understanding.

Participants were given a timed break at the end of each block and asked to continue on.

\subsection{Electrophysiological recording}

The continuous scalp electroencephalogram (EEG) was recorded from 19 sites (Fp1, Fp2, F3, F4, F7, F8, Fz, C3, C4, Cz, P3, P4, Pz, T3, T4, T5, T6, O1, O2) using an electrode cap containing tin electrodes fitted according to the international 10-20 system (Jasper, 1958). A ground electrode located between Fpz and Fz, and all electrodes were referenced to linked ears. EOG was measured vertically with two tin cup electrodes, $1 \mathrm{~cm}$ above and below the left eye. Impedance was kept below $3 \mathrm{k} \Omega$ for EOG and reference electrodes, and below $5 \mathrm{k} \Omega$ for cap electrodes. EEG and EOG signals were amplified 19 times and sampled at $500 \mathrm{~Hz}$, with bandpass down $3 \mathrm{db}$ at 0.1 and $100 \mathrm{~Hz}$ via a NuAmps system (Compumedics Limited,

\footnotetext{
${ }^{1}$ Although participants were instructed to rate their perceived effort irrespective of their task performance, knowledge of their performance may still have influenced their ratings.
} 
Melbourne, Australia). Prior to processing, the EEG data were digitally filtered using a low-pass filter $3 \mathrm{db}$ down at $30 \mathrm{~Hz}$.

\subsection{Skin Conductance recording}

Electrodermal activity was recorded using two $\mathrm{Ag} / \mathrm{AgCl}$ electrodes placed on the distal phalanges of the third and fourth digits of the left hand. Recording electrodes were filled with electrode paste $(0.05 \mathrm{M} \mathrm{NaCl}$ in an inert viscous ointment base) and secured using velcro straps and tape. A constant voltage device (UFI Bioderm model 2701) set at $0.5 \mathrm{~V}$ was used. This system separately recorded tonic DC-coupled SCL, AC-coupled skin conductance response (SCR), measured in microsiemens $(\mu \mathrm{S})$ but only SCL is reported here.

\subsection{Data Quantification}

The ERP epoch was defined as $100 \mathrm{~ms}$ pre-stimulus to $900 \mathrm{~ms}$ post-stimulus onset. Epochs were excluded if they contained activity greater than $\pm 100 \mu \mathrm{V}$ at any non-frontal site. EOG artefact reduction was carried out based on vertical EOG (Semlitsch et al., 1986). ERPs were averaged across epochs for correct responses only. This resulted in a minimum of 18 artefactand-error-free Nogo trials being included in each average. To ensure compatibility withinsubjects, the number of epochs available for averaging was determined for Nogo stimuli initially, with Go epochs restricted to the same number, being selected randomly from the total available. Grand average ERP waveforms for Go and Nogo stimuli were displayed in order to define the components latency range. Latency at all sites was locked to the peak latency at the site of maximum amplitude, with amplitude for all 9 electrodes taken at the same post-stimulus latency (Picton et al., 2000; Spencer et al., 2001). ERP component peaks were quantified using automatic peak-picking software which identified the largest positive or negative deflections within the predefined latency range, relative to the $100 \mathrm{~ms}$ pre-stimulus baseline period. Peak latency ranges and sites were as follows: N1 (100 -160 ms Fz), P2 (180-240 ms Pz), N2 (200$280 \mathrm{~ms} \mathrm{Fz}$ ), P3 (280-520 ms Pz). Skin conductance level was taken as the average value (in $\mu \mathrm{S}$ ) for each $30 \mathrm{sec}$ period over the $3.5 \mathrm{~min}$ duration of each block of the Go/Nogo task.

\subsection{Statistical analyses}

The error rate (Go omission errors, Go RTD errors and Nogo errors) was calculated as the number of incorrect responses divided by the total number of trials. The Go/Nogo performance data were subject to Condition [Low (L) vs. Medium (M) vs. High (H)] x Time [Block 1 (b1) vs. Block 4 (b4) vs. Block 8 (b8)] mixed design ANOVAs, with repeated measures on the 
within-subjects factors. Planned orthogonal contrasts were used to analyse differences within Time and between conditions using Linear (b1 vs. b8) and Quadratic (mean of b1/b8 vs. b4) contrasts.

Primary analyses of the ERP data were restricted to the sites F3, Fz, F4, C3, Cz, C4, P3, Pz and P4. Go and Nogo data were subject to a Condition (L vs. M vs. H) x Lateral (Left vs. Midline vs. Right) x Sagittal (Frontal vs. Central vs. Parietal) x Stimulus (Go vs. Nogo) x Time (b1 vs. b4 vs. b8) ANOVAs. Planned orthogonal contrasts, which allow insight into training-related changes in the topographic distribution of each component, were performed on the withinsubjects factors. The Sagittal factor compared the frontal region (mean of F3, Fz and F4) with the posterior region (mean of $\mathrm{P} 3, \mathrm{Pz}$ and $\mathrm{P} 4$ ), and their mean with the central region (mean of $\mathrm{C} 3, \mathrm{Cz}$ and $\mathrm{C} 4$ ). The Lateral factor compared activity in the left hemisphere (mean of F3, C3 and P3) with that in the right hemisphere (mean of F4, C4 and P4), and their mean with the midline region (mean of $\mathrm{Fz}, \mathrm{Cz}$ and $\mathrm{Pz}$ ). Finally, the Time factor compared block 1 to block 8 (Linear contrast), and their mean with block 4 (Quadratic). The analyses for component peak latency excluded site contrasts. As these contrasts were planned with no more of them than the degrees of freedom for each effect, no Bonferroni type adjustment to $\alpha$ were necessary (Tabachnick and Fidell, 1996). Also, single degrees of freedom contrasts are not affected by violations of symmetry assumptions common in repeated measures analyses, and thus do not require Greenhouse-Geisser-type corrections. As these analyses are carried out over a substantial number of variables, each may be considered to constitute a separate experiment. It should be noted that this increases the frequency of type 1 errors, however, as this is an increase in frequency, rather than probability, it cannot be 'controlled' by adjustment of alpha levels (Howell, 2009). All ERP statistics have $(1,58)$ degrees of freedom unless otherwise indicated. Outliers in the data (i.e. values exceeding \pm 2.5 standard deviations from the mean) were corrected for by replacing with the series mean $(<1.1 \%$ for any task performance or ERP variable). Data were normalised using the vector scaling method (McCarthy and Wood, 1985), and only interactions with topography that remained significant in the normalised data are reported here.

\section{Results}

\subsection{Manipulation check, perceived effort and SCL}

Participants' perceived effort was greater in the High $(M=3.87)$ than the Medium $(M=3.57)$ and Low $(\mathrm{M}=3.20)$ conditions (i.e. $\mathrm{H}>\mathrm{M}>\mathrm{L}$; Linear: $F=6.13, p=.016, \eta 2=.096$ ). 
Similarly, there were incremental increases in Nogo errors (see Figure 1d) with each decrease in RTD, with the greatest overall percentage of errors in the High condition (Linear: $F=77.70, p$ $<.001, \eta 2=.577)$. Combined, these results suggest that three task difficulty levels were established, with greater perceived effort and declines in inhibitory performance with shorter RTDs. Across the session, the Time main effect (Linear: $F=.031, p=.862$ ) and the Time $\mathrm{x}$ Condition interaction (Linear: $F=.031, p=.970$ ) for perceived effort were not significant. SCL increased from the beginning $(11.6 \mu \mathrm{S})$ to the end of the training session $(13.1 \mu \mathrm{S}$; Linear: $F=$ $23.20, p<.001, \eta 2=.289$ ), but this effect did not differ between conditions (Linear: $F=2.08, p$ $=.134)$.

\subsection{Task Performance}

As seen in Figure 1a, Go reaction time (RT) decreased with training showing the largest decline in the Low condition (Linear: $F=3.32, p=.043, \eta 2=.032$ ). Go omission (Linear, $F=3.41, p$ $=.040, \eta 2=.051$ ) and Go RTD errors (Linear, $F=20.84, p<.001, \eta 2=.198$; Quad: $F=5.87, p$ $=.005, \eta 2=.059$ ) decreased early in the session (i.e. by block 4 ) for the Medium and High conditions - with the greatest declines for the High condition (see Figure 1b and c). By contrast, Go RTD errors and Go omission errors appeared to be at ceiling for the Low condition and did not modulate over the course of training.

As seen in Figure 1d, Nogo errors showed different training effects with task difficulty. While Nogo errors increased sharply from block 1 to block 4 and plateaued thereafter for High condition, the Medium and Low condition remained relatively stable across the session (Linear: $F=4.04, p=.023, \eta 2=.124$; Quadratic: $F=4.84, p=.011, \eta 2=.145)$. A further within Condition analysis of Nogo errors confirmed no change for the Low (Linear: $F=2.05, p=.169$ ) or Medium condition (Linear: $F=0.24, p=.632$ ), but a significant increase for the High (Linear: $F=4.39, p=.050, \eta 2=.188$ ). To clarify whether the facilitation of Go RT with training resulted in a speed-accuracy trade-off (SAT), we correlated the training-related change (i.e. b8 - b1) in Go RT and Nogo errors separately for each condition. This analyses was not significant for either the Low $(r=-.104, p=.664)$ or Medium condition $(r=-.074, p=.758)$, but it was for the High $(r=-.467, p=.038)$. Given that Nogo errors remained stable for the Low and Medium conditions, declines in Go RT with training represent an improvement in behavioural Go/Nogo proficiency. This result is similar to Manuel et al. (2010). 

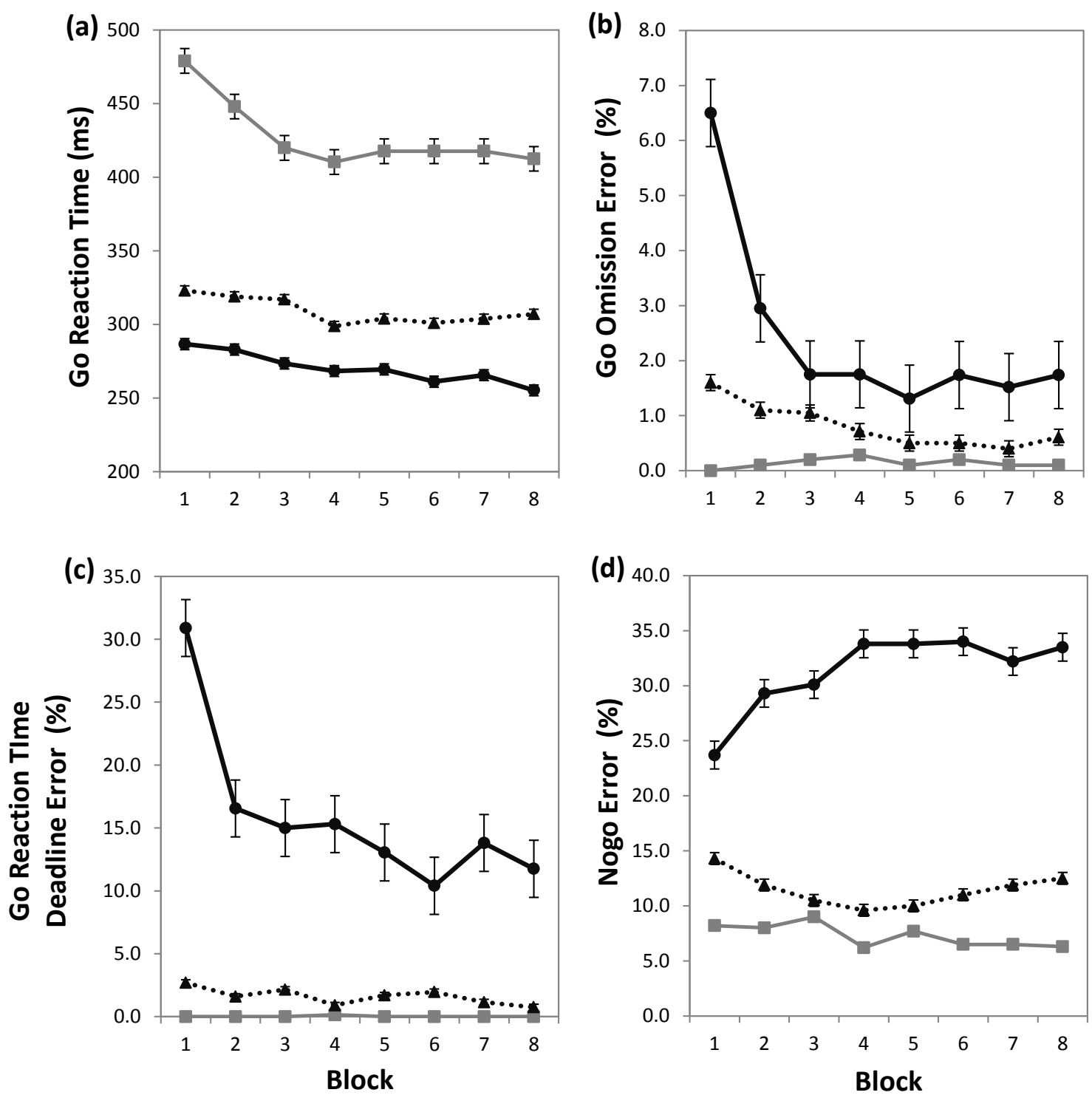

\section{$\longrightarrow$ Low $\cdots \ldots \cdot$ Medium $\rightarrow$ High}

Figure 1. Task performance indices for each difficulty condition over the training session including (a) Go reaction time, (b) Go Omission, (c) Go reaction time deadline, and (d) Nogo errors. Error bars represent standard error of the mean. Note: Data for all eight blocks is included for display purposes, but only block 1, 4 and 8 were considered in the statistical analyses. 


\subsection{Event- related Potentials}

Figure 2 presents grand mean ERPs to Go/Nogo stimuli across conditions (top left panel) and for each condition separately (remaining three panels) for blocks 1, 4 and 8. ERP latency data is presented in Table 1. As the primary aim of this study was the effect of varying task difficulty on the training of inhibitory control, and as a large body of literature has been devoted to descriptions of the topography of the various ERP components, reporting of the results will focus on effects and interactions involving Time and Condition.

The waveforms were characterised by an N1-P2 complex, most apparent at frontal and central sites, followed by an $\mathrm{N} 2$ component at about $270 \mathrm{~ms}$ primarily in the frontocentral region. The P3 is evident as a large positivity which peaks approximately $300-400 \mathrm{~ms}$ post-stimulus and is largest parietally. 


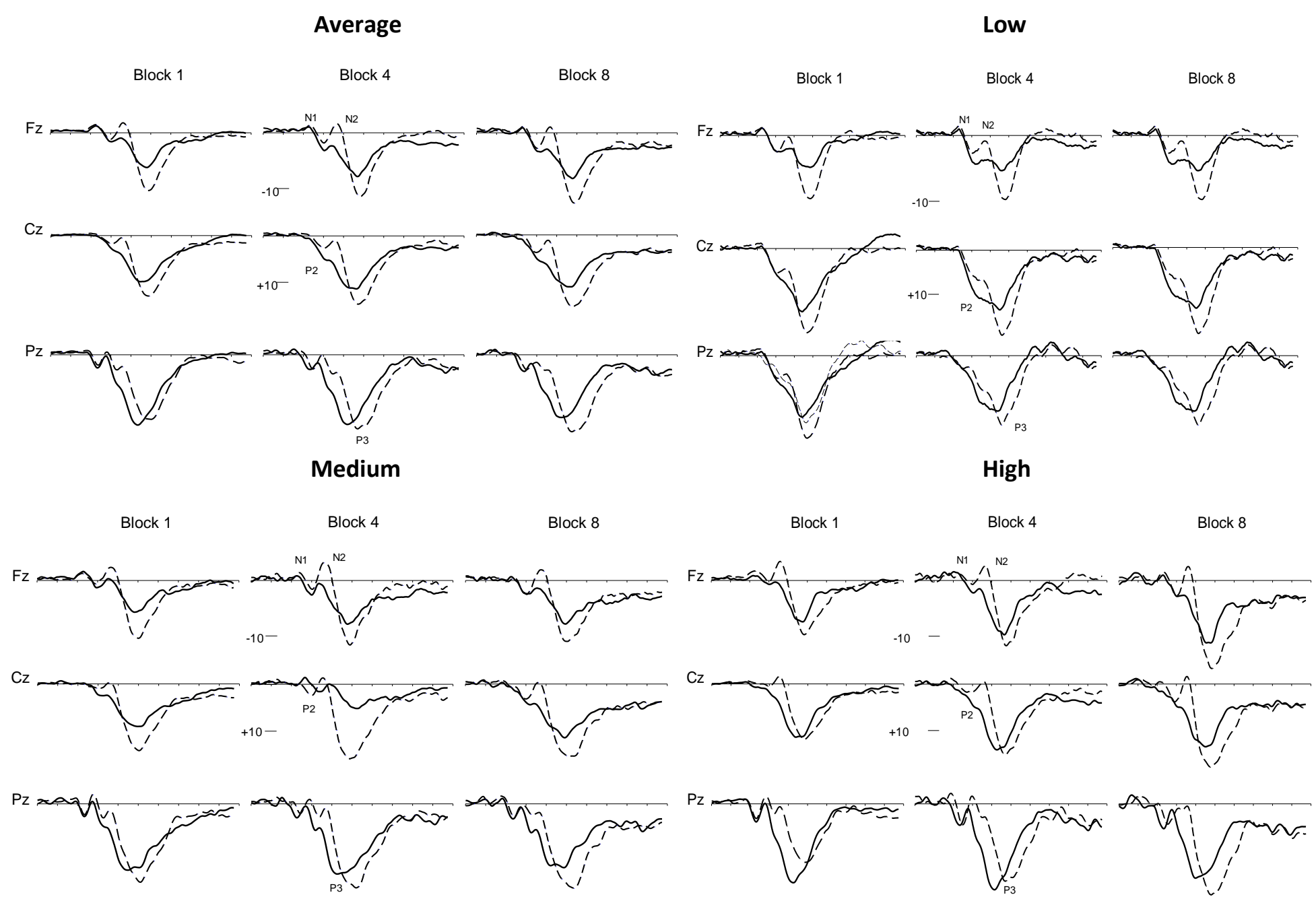

Figure 2. Grand mean ERPs for blocks 1, 4 and 8 to Go (solid line) and Nogo (dashed line) across condition (top left panel) and for each task difficulty condition separately (remaining three panels). ERPs are shown at three midline sites only. Note: $\mathrm{x}$-axis ticks $=100 \mathrm{~ms}$; stimulus onset at $\mathrm{y}$-axis (scale: \pm 10 $\mu \mathrm{V}$ ) shown at $\mathrm{Cz}$. 
Table 1. Mean latency (in ms) for Go/Nogo stimuli between each task difficulty condition for blocks 1,4 and 8 (Standard deviations in parentheses).

\begin{tabular}{|c|c|c|c|c|c|c|c|c|c|}
\hline \multirow[t]{2}{*}{ ERP } & \multicolumn{3}{|c|}{ Block 1} & \multicolumn{3}{|c|}{ Block 4} & \multicolumn{3}{|c|}{ Block 8} \\
\hline & Low & Medium & High & Low & Medium & High & Low & Medium & High \\
\hline \multicolumn{10}{|l|}{ Go } \\
\hline N1 & $\begin{array}{l}139.0 \\
(12.7)\end{array}$ & $\begin{array}{l}146.2 \\
(20.0)\end{array}$ & $\begin{array}{l}142.9 \\
(25.5)\end{array}$ & $\begin{array}{l}134.3 \\
(32.1)\end{array}$ & $\begin{array}{l}142.8 \\
(20.3)\end{array}$ & $\begin{array}{l}127.8 \\
(23.4)\end{array}$ & $\begin{array}{l}129.8 \\
(21.0)\end{array}$ & $\begin{array}{l}142.7 \\
(25.1)\end{array}$ & $\begin{array}{l}130.6 \\
(28.2)\end{array}$ \\
\hline P2 & $\begin{array}{l}233.3 \\
(22.5)\end{array}$ & $\begin{array}{l}221.3 \\
(16.8)\end{array}$ & $\begin{array}{l}225.9 \\
(23.3)\end{array}$ & $\begin{array}{l}226.7 \\
(37.4)\end{array}$ & $\begin{array}{l}225.0 \\
(21.2)\end{array}$ & $\begin{array}{l}212.4 \\
(37.1)\end{array}$ & $\begin{array}{l}214.4 \\
(30.2)\end{array}$ & $\begin{array}{l}233.2 \\
(31.6)\end{array}$ & $\begin{array}{l}209.1 \\
(46.3)\end{array}$ \\
\hline N2 & $\begin{array}{l}282.8 \\
(23.3)\end{array}$ & $\begin{array}{l}267.1 \\
(19.1)\end{array}$ & $\begin{array}{l}262.0 \\
(18.8)\end{array}$ & $\begin{array}{l}265.9 \\
(46.0)\end{array}$ & $\begin{array}{l}260.8 \\
(21.2)\end{array}$ & $\begin{array}{l}243.2 \\
(35.8)\end{array}$ & $\begin{array}{l}254.7 \\
(38.3)\end{array}$ & $\begin{array}{l}272.8 \\
(37.7)\end{array}$ & $\begin{array}{l}264.1 \\
(40.6)\end{array}$ \\
\hline P3 & $\begin{array}{l}368.9 \\
(40.6)\end{array}$ & $\begin{array}{l}398.1 \\
(34.2)\end{array}$ & $\begin{array}{l}355.7 \\
(29.6)\end{array}$ & $\begin{array}{l}350.3 \\
(46.7)\end{array}$ & $\begin{array}{l}358.0 \\
(48.8)\end{array}$ & $\begin{array}{l}337.1 \\
(27.3)\end{array}$ & $\begin{array}{l}324.1 \\
(34.4)\end{array}$ & $\begin{array}{l}378.1 \\
(49.9)\end{array}$ & $\begin{array}{l}352.8 \\
(42.7)\end{array}$ \\
\hline \multicolumn{10}{|l|}{ Nogo } \\
\hline $\mathrm{N} 1$ & $\begin{array}{l}141.0 \\
(20.4)\end{array}$ & $\begin{array}{l}144.9 \\
(15.6)\end{array}$ & $\begin{array}{l}149.1 \\
(21.5)\end{array}$ & $\begin{array}{l}136.1 \\
(25.0)\end{array}$ & $\begin{array}{l}141.3 \\
(17.1)\end{array}$ & $\begin{array}{l}135.7 \\
(26.4)\end{array}$ & $\begin{array}{l}133.0 \\
(16.7)\end{array}$ & $\begin{array}{l}145.2 \\
(22.6)\end{array}$ & $\begin{array}{l}130.1 \\
(16.7)\end{array}$ \\
\hline $\mathrm{P} 2$ & $\begin{array}{l}232.2 \\
(25.8)\end{array}$ & $\begin{array}{l}226.6 \\
(14.6)\end{array}$ & $\begin{array}{l}221.0 \\
(16.7)\end{array}$ & $\begin{array}{l}223.2 \\
(28.1)\end{array}$ & $\begin{array}{l}229.2 \\
(20.3)\end{array}$ & $\begin{array}{l}205.4 \\
(27.4)\end{array}$ & $\begin{array}{l}233.2 \\
(23.5)\end{array}$ & $\begin{array}{l}226.3 \\
(23.8)\end{array}$ & $\begin{array}{l}208.1 \\
(18.3)\end{array}$ \\
\hline N2 & $\begin{array}{l}285.8 \\
(22.4)\end{array}$ & $\begin{array}{l}274.4 \\
(18.8)\end{array}$ & $\begin{array}{l}270.1 \\
(17.5)\end{array}$ & $\begin{array}{l}266.6 \\
(24.8)\end{array}$ & $\begin{array}{l}271.9 \\
(17.7)\end{array}$ & $\begin{array}{l}251.8 \\
(32.8)\end{array}$ & $\begin{array}{l}274.1 \\
(24.4)\end{array}$ & $\begin{array}{l}272.8 \\
(22.4)\end{array}$ & $\begin{array}{l}257.1 \\
(15.4)\end{array}$ \\
\hline P3 & $\begin{array}{l}382.7 \\
(35.2)\end{array}$ & $\begin{array}{l}418.6 \\
(21.0)\end{array}$ & $\begin{array}{l}400.4 \\
(22.7)\end{array}$ & $\begin{array}{l}366.2 \\
(39.9)\end{array}$ & $\begin{array}{l}418.4 \\
(34.5)\end{array}$ & $\begin{array}{l}392.6 \\
(46.8)\end{array}$ & $\begin{array}{l}379.9 \\
(42.5)\end{array}$ & $\begin{array}{l}419.3 \\
(30.3)\end{array}$ & $\begin{array}{l}383.9 \\
(41.6)\end{array}$ \\
\hline
\end{tabular}




\subsection{N1}

N1 latency (Mean $138.5 \mathrm{~ms}$ ) declined linearly across the training session (see Table 1; Linear: $F$ $=13.94, p<.001, \eta 2=.196$ ). A Time $\mathrm{x}$ Condition effect approached significance (Linear: $F=$ $3.11, p=.052, \eta 2=.098)$ indicating that the $\mathrm{N} 1$ latency reduction was greater for the High (b1 vs. b8 diff.: $15.5 \mathrm{~ms}$ ) than the Low (b1 vs. b8 diff.: $8.6 \mathrm{~ms}$ ) and Medium conditions (b1 vs. b8 diff.: $1.5 \mathrm{~ms})$.

Table 2 summarises the following effects and provides means. N1 amplitude reduced linearly from the beginning until the end of the session. Interestingly, training differentially modulated Go/Nogo N1 amplitudes between conditions (Stimulus x Condition x Time interaction), in that a Go > Nogo N1 effect, which was larger for the Low than Medium condition in block 1, reduced across the training session to be almost equipotential for both conditions by block 8; contrasting with the High condition, that displayed a training-related reduction in the Nogo relative to Go N1 (see Figure 3).

Table 2. Significant results for the N1 and P2 components.

\begin{tabular}{|c|c|c|c|c|c|}
\hline Measure & Effect & Contrast & Details & $\mathbf{F}$ & $\eta^{2}$ \\
\hline \multirow[t]{5}{*}{$\mathrm{N} 1$} & $\mathrm{~T}$ & Linear: b1 vs. b8 & -1.0 vs. -0.3 & $6.95^{*}$ & .063 \\
\hline & & Quadratic: b4 vs. b1/b8 & -0.3 vs. -0.6 & $3.65^{*}$ & .024 \\
\hline & T $x$ Stim $x$ Cond & Linear: Go vs. Nogo & Low: b1, -0.9 vs. $-0.1 ; b 8,-0.1$ vs. -0.2 & & \\
\hline & & & Medium: b1, -1.2 vs. $-1.1 ; b 8,-0.4$ vs. -0.2 & & \\
\hline & & & High: b1 -0.7 vs. $-1.6 ; b 8,-0.9$ vs. 0.0 & $3.83 * *$ & .048 \\
\hline \multirow[t]{8}{*}{$\mathrm{P} 2$} & Stim & Go vs. Nogo & 5.4 to 3.5 & $37.56^{* * *}$ & .375 \\
\hline & $\mathrm{T}$ & Linear: b1 vs. b8 & 3.6 vs. 5.1 & $7.60 * *$ & .073 \\
\hline & T x S x Stim x Cond & Linear: $f$ vs. $p$ & Low: b1, Go, 3.9 to 6.7 vs. Nogo, 3.1 to 6.2 ; & & \\
\hline & & & b8, Go, 5.4 to 7.6 vs. Nogo, 3.7 to 8.2 & & \\
\hline & & & Medium: b1, Go, 2.2 to 4.9 vs. Nogo, 0.6 to 4.0 ; & & \\
\hline & & & b8, Go, 3.6 to 7.5 vs. Nogo, 2.2 to 3.5 & & \\
\hline & & & High: b1, Go, 1.7 to 7.3 vs. Nogo, 1.4 to 4.2 ; & & \\
\hline & & & b8: Go, 3.5 to 6.7 vs. Nogo, 2.8 to 2.1 & $4.37^{*}$ & .022 \\
\hline
\end{tabular}

$*=<.05, * *=<.01, * * *=<.001$

Note: For this and subsequent tables, details column represents mean amplitude in $\mu \mathrm{V}$. Cond, Condition: Low/Medium/High task difficulty. Low, Low task difficulty condition. Medium, Medium difficulty condition, High, High difficulty condition. Stim, Stimulus type: Go/NoGo. T, Time; Linear: Linear contrast comparing block 1 to block 8; Quadratic: Quadratic contrast comparing the average of block 1/8 and block4; b1, block 1; b1/b8, average of block 1 and 8; b8, block 8. Sagittal (S) abbreviations: f, mean frontal (F3, Fz, F4); p, mean parietal (P3, Pz, P4); c, mean central (C3, Cz, C4); f/p, mean of frontal and parietal (F3, Fz, F4, P3, Pz, P4). 

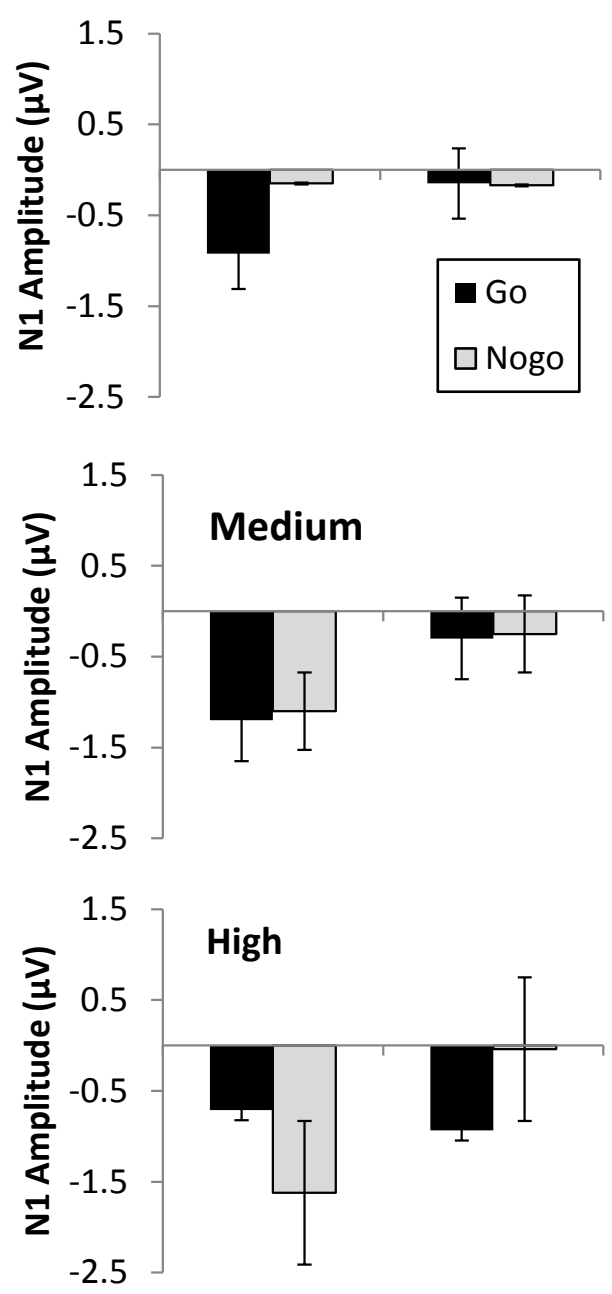

\section{Block 1 Block 8}

Figure 3. Stimulus x Condition x Time interaction for Go and Nogo N1 amplitude.

\subsection{P2}

P2 latency (Mean $222.7 \mathrm{~ms}$ ) declined across the training session (Table 1; Linear: $F=6.26, p$ $=.015, \eta 2=.053$ ). This reduction was greater for the High (b1 vs. b8 diff.: $21.4 \mathrm{~ms}$ ) than Low condition (b1 vs. b8 diff.: $6.8 \mathrm{~ms}$ ), in contrast to the Medium condition that displayed a slightly longer $\mathrm{P} 2$ by the end of the session (b1 vs. b8 diff.: $+2.2 \mathrm{~ms}$; Linear: $F=3.96, p=.025, \eta 2$ $=.059)$.

P2 showed a Go > Nogo effect (see Table 2). The amplitude of the P2 increased linearly with training. As evidenced by significant Time x Sagittal x Stimulus x Conditions interactions, Go and Nogo P2 topography differed between conditions: for the Go P2, a central > frontal/parietal effect increased linearly with task difficulty (i.e. $\mathrm{H}>\mathrm{M}>\mathrm{L}$ ), suggestive of an anterior shift of 


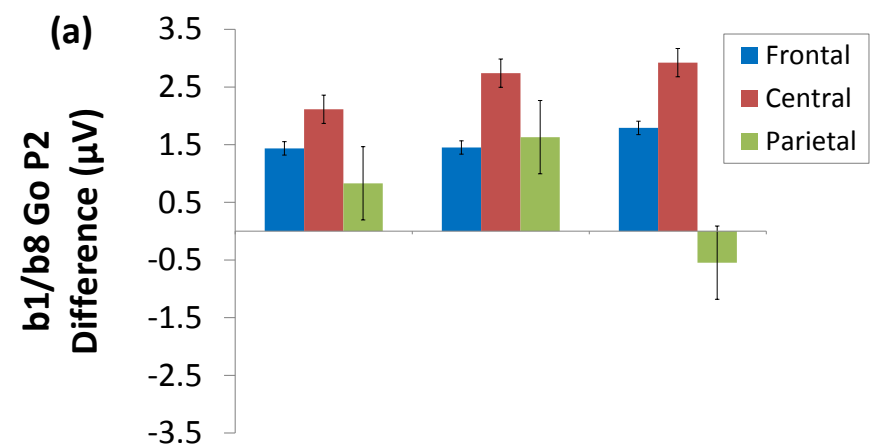

(b)

b1/b8 Go P2 Difference

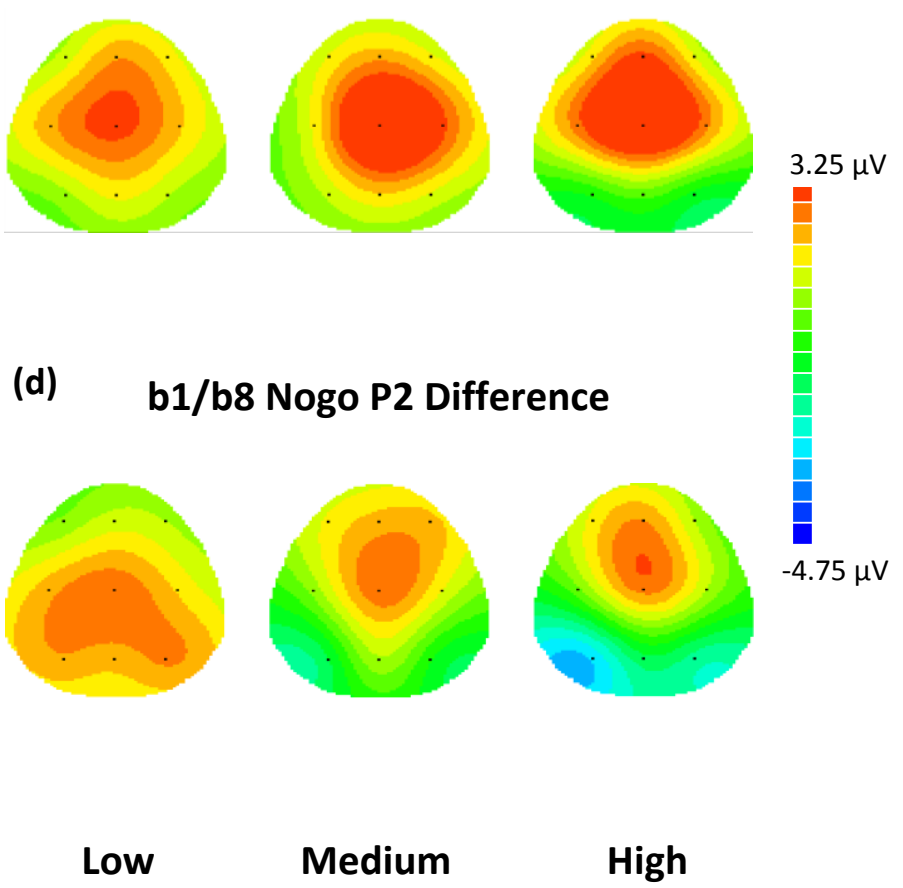

Figure 4. Mean change in the (a) Go P2 and (c) Nogo P2 from block 1 to block 8 across the Sagittal dimension. Error bars represent standard error of the mean. Topographic maps for the mean change in voltage distribution from block 1 to block 8 for the (b) Go P2 and (d) Nogo P2. Scale values represent the ends of the colour scale in $\mu \mathrm{V}$ for each component. Darkest blue = negativity, red = positivity. 
the Go P2 focus with training, particularly apparent in the Medium and High conditions (Figure 4a and b). For Nogo P2 the Medium and High conditions displayed a more anterior Nogo P2 with training, the Low condition showed the opposite pattern, with enhanced Nogo P2 activity at centroparietal regions (Figure $4 \mathrm{c}$ and d). Simple effects analyses confirmed a significant Sagittal x Time x Condition effect to Go (Quad: $F=3.60, p=.032, \eta 2=.011$ ) and Nogo stimuli (Linear: $F=5.58, p=.006, \eta 2=.055$ ).

\section{$3.6 \mathrm{~N} 2$}

N2 (Mean $266.5 \mathrm{~ms}$ ) peaked later to Nogo $(269.2 \mathrm{~ms})$ than Go stimuli $(263.7 \mathrm{~ms} ; F=6.09, p$ $=.017, \eta 2=.097)$. Linear $(F=7.96, \mathrm{p}=.007, \eta 2=.122)$ and quadratic $(\mathrm{F}=8.51, p=.005, \eta 2$ $=.130)$ effects indicated that while the $\mathrm{N} 2$ latency declined rapidly from block 1 (273.7 $\mathrm{ms})$ to block 4 (260.1 ms), it began to increase slightly by block 8 (265.9 ms). Training effects for N2 latency also differed between conditions, showing a large decline for the Low (b1 vs. b8 diff.: $19.9 \mathrm{~ms}$ ), but little change for the High (b1 vs. b8 diff: $5.4 \mathrm{~ms}$ ), and Medium conditions (b1 vs. b8 difference: $2.0 \mathrm{~ms} ; F=5.49, p=.007, \eta 2=.161$ ).

Globally, N2 amplitude was larger to Nogo than Go stimuli (see Table 3 for effect summaries and means). Overall, N2 amplitude decreased across the training session. Moreover, a Time $\mathrm{x}$ Stimulus interaction showed that the Nogo > Go N2 effect increased linearly from block 1 to block 8. However, inspection of the means (see Table 3 and Figure 5) shows that it was the Go N2, and not the Nogo N2 that declined across the session. Follow-up analyses confirmed a significant reduction across the session for the Go N2 (Linear: $(F=11.57, p=.001, \eta 2=.095)$, but not the Nogo N2 (Linear: $F=0.00, p=.995$ ). Against predictions, these effects did not differ between conditions. 
Table 3. Significant results for the N2 and P3 components.

\begin{tabular}{|c|c|c|c|c|c|}
\hline Measure & Effect & Contrast & Details & $\mathbf{F}$ & $\eta^{2}$ \\
\hline \multirow[t]{5}{*}{$\mathrm{N} 2$} & Stim & Go vs. Nogo & 4.1 vs. 0.0 & $133.00 * * *$ & .634 \\
\hline & $\mathrm{T}$ & Linear: b1 vs. b8 & 1.6 vs. 2.6 & $4.36 * *$ & .043 \\
\hline & Tx Stim & Linear: b1 vs. b8 & Go, 3.3 vs. $0.0 ;$ Nogo: 5.2 vs. 0.0 & $9.88 * *$ & .071 \\
\hline & Tx S x Stim & Linear: c vs. f/p & b1: Go, 3.6 vs. 3.1, Nogo, 0.1 vs. -0.1 & & \\
\hline & & & b8: Go, 6.2 vs. 4.7, Nogo, 0.0 vs. -0.1 & $20.7 * * *$ & .030 \\
\hline \multirow[t]{18}{*}{ P3 } & Stim & Go vs. Nogo & 12.7 vs. 15.7 & $36.71^{* * *}$ & .378 \\
\hline & S x Stim & f vs. $p$ & Go: 8.4 vs. $15.2 ;$ Nogo: 12.7 vs. 16.4 & $57.28 * * *$ & .405 \\
\hline & & c vs. $\mathrm{f} / \mathrm{p}$ & Go: 14.4 vs. $11.8 ;$ Nogo: 17.9 VS. 14.6 & $13.89^{* * *}$ & .029 \\
\hline & $\mathrm{T}$ & b1 vs. b8 & 13.0 vs. 15.0 & $15.23 * * *$ & .129 \\
\hline & T x Sim x Cond & Go vs. Nogo & Low: b1, 9.9 vs. $12.9 ;$ b8, 13.1 vs. 14.9 & & \\
\hline & & & Medium: b1, 12.5 vs. $15.6 ;$ b8, 11.8 vs. 16.7 & & \\
\hline & & & High: b1, 13.7 vs. $13.6 ; 14.1$ vs. 19.7 & $6.39 *$ & .049 \\
\hline & T x S x Stim x Cond & Linear: $\mathrm{f}$ vs. $\mathrm{p}$; & Low: b1,Go, 6.4 vs. $12.2 ; \mathrm{Nog}_{0}, 9.8$ vs. 13.9 & & \\
\hline & & & b8, Go, 15.4 vs. $11.9 ;$ Nogo, 12.6 vs. 15.0 & & \\
\hline & & & Medium: b1,Go, 8.3 vs. $15.9 ;$ Nogo, 12.1 vs. 17.0 & & \\
\hline & & & b8, Go, 7.7 vs. $14.4 ;$ Nogo, 13.3 vs. 17.3 & & \\
\hline & & & High: b1,Go, 9.3 vs. $16.6 ; \mathrm{Nog}_{0}, 11.5$ vs. 14.3 & & \\
\hline & & & b8, Go, 10.5 vs. $15.6 ;$ Nogo, 17.3 vs. 19.5 & $4.95^{*}$ & .028 \\
\hline & & Quadratic: c vs. f/p & Low: b1,Go, 11.2 vs. $9.3 ; \mathrm{Nog}_{0}, 17.8$ vs. 14.5 & & \\
\hline & & & b8, Go, 15.4 vs. $11.9 ;$ Nogo, 17.0 vs. 13.8 & & \\
\hline & & & $\begin{array}{l}\text { Medium: b1,Go, } 13.5 \text { vs. } 12.1 ; \text { Nogo, } 12.1 \text { vs. } 17.0 \\
\text { b8, Go, } 13.4 \text { vs. } 11.0 ; \text { Nogo, } 19.4 \text { vs. } 15.3\end{array}$ & & \\
\hline & & & High: b1,Go, 15.2 vs. $13.0 ;$ Nogo, 15.1 vs. 12.9 & & \\
\hline & & & b8, Go, 16.3 vs. $13.1 ;$ Nogo, 22.3 vs. 18.4 & $3.92 *$ & .007 \\
\hline
\end{tabular}

$*=<.05, * *=<.01, * * *=<.001$ 


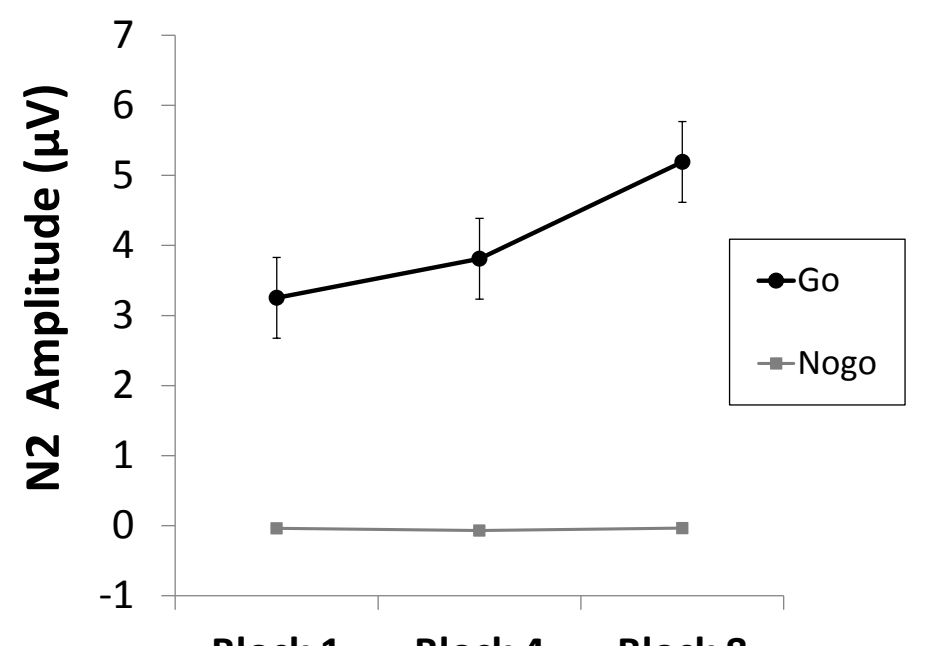

Figure 5. Go and Nogo N2 amplitude across the training session.

Error bars represent standard error of the mean.

\subsection{P3}

The P3 (Mean $376.9 \mathrm{~ms})$ peaked later for Nogo $(395.8 \mathrm{~ms})$ than Go stimuli $(358.1 \mathrm{~ms} ; F=$ $64.64, p<.001, \eta 2=.531$ ). While Nogo P3 latency remained relatively stable across the session for each condition, Go P3 latency showed the greatest training-related declines for the Low (b1 vs. b8 diff: $44.7 \mathrm{~ms}$ ) compared to the Medium (b1 vs. b8 diff: $19.9 \mathrm{~ms}$ ) and High conditions (b1 vs. b8 diff: $2.9 \mathrm{~ms} ; F=4.68, p=.014, \eta 2=.138)$.

The P3 was larger to Nogo than Go stimuli (see Table 3 for effect summaries and means), with a smaller parietal $>$ frontal gradient (parietal vs. frontal difference: Nogo $3.7 \mu \mathrm{V}$, Go $6.8 \mu \mathrm{V}$ ) and an increased central > frontal/parietal effect in Nogo compared to Go stimuli (central vs. frontal/parietal difference: Nogo $3.3 \mu \mathrm{V}$, Go $2.6 \mu \mathrm{V}$ ). These effects highlighted the anteriorisation of $\mathrm{P} 3$ to Nogo relative to Go stimuli.

P3 amplitude increased with training. The Nogo > Go P3 effect (across the scalp) was reduced for the Low condition by block 8 (Go vs. Nogo diff.: b1, 3.0 vs. b8, $1.8 \mu \mathrm{V}$ ), while the High condition (Go vs. Nogo diff.: b1, 0.0 vs. b8, $5.6 \mu \mathrm{V}$ ) showed a significantly larger traininginduced increase in the Nogo > Go P3 effect than the Medium condition (Go vs. Nogo diff.: b1, 3.1 vs. b8, $4.9 \mu \mathrm{V}$ ). Time $\mathrm{x}$ Sagittal $\mathrm{x}$ Stimulus $\mathrm{x}$ Condition interactions indicated that this effect was most apparent over central regions (Figure 6), as indicated by more anterior Nogo than Go P3 for the High than the Medium and Low conditions. 
Block 1
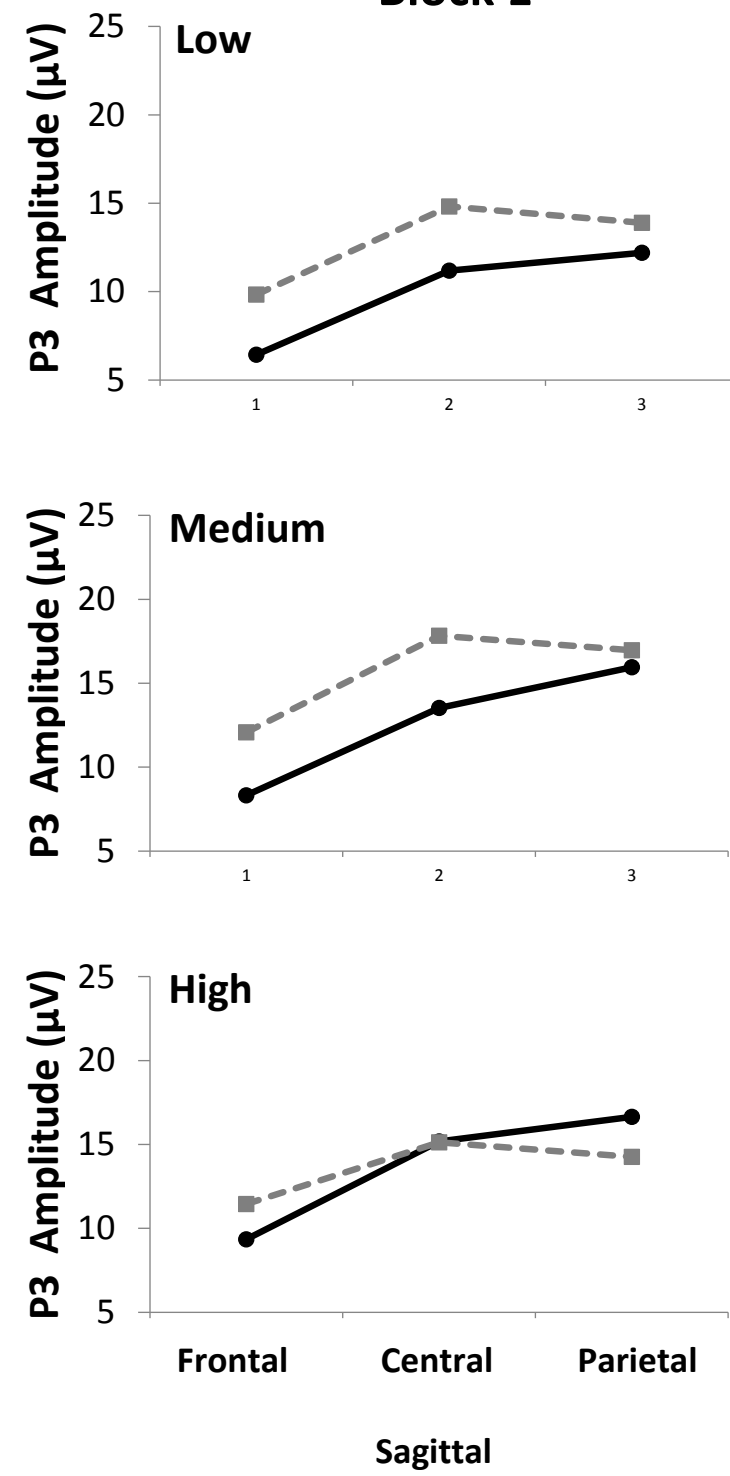

$$
\rightarrow-G o-n \cdot N o g o
$$

Figure 6. Time x Stimulus x Sagittal x Condition interaction for P3 amplitude. 


\section{Discussion}

The questions of how to reliably attain training-induced improvements in inhibitory control and the supporting neural mechanisms remains unresolved. Therefore, using task performance and neural markers of inhibitory processing, the primary focus of the present research was to investigate the effect of varying task difficulty during short-term training of the Go/Nogo task. In addition, we also aimed to determine whether the early evoked potentials and state differences (as indexed by task-related arousal and perceived effort) would be modulated by training.

\subsection{Task Performance}

Performance findings revealed that the Go/Nogo training was significantly influenced by variations in task difficulty. Both the Low and Medium conditions showed considerable reductions in Go RT along with no change in Nogo accuracy; suggesting a training-related improvement in inhibitory control, given that accuracy was maintained in the context of faster responding (for a similar finding see Manuel et al., 2010). The relationship between fast Go responding and an increased requirement for inhibition on Nogo trials is well-established (Band et al., 2003; Falkenstein et al., 2000; Falkenstein et al., 1999; Jodo and Kayama, 1992; Lindqvist and Thorell, 2009; Manuel et al., 2010; Smith et al., 2006). When responses grow progressively faster on average, the relative strength of inhibition must increase in order to overcome the fast Go response (Smith et al., 2006). Moreover, given that Go RT was much faster overall for the Medium than Low condition (in addition to large improvements in Go RTD errors and Omission errors for the Medium condition), it appears that moderate task difficulty leads to greater enhancements in Go/Nogo proficiency. Together with the finding that the High condition showed a significant decline in inhibitory performance, these findings are in line with previous behavioural reports suggesting that learning is likely to be enhanced when a task remains relatively challenging but not overly difficult (Ahissar and Hochstein, 2004), and provide novel evidence for the appropriate use of RTDs in optimising the short-term training of inhibitory control.

\subsection{Task-related arousal}

In order to investigate the role of state factors during the training session we examined taskrelated arousal and perceived effort. While perceived effort did not significantly vary over the training session, all three conditions showed a linear increase in arousal. Based on their review of this literature, Dawson et al. (1990) have suggested that elevations in skin conductance 
reflects the effortful mobilisation of mental resources directed towards a task (for similar conclusions see Johnstone et al., 2009; Larue et al., 2011; Naccache et al., 2005). The enhancement of task-related arousal could therefore be related to the increased efforts of participants to maintain an alert state throughout the training session irrespective of task difficulty condition. However, it should be noted that other factors including mood and fatigue have been shown to influence not only SCL (e.g. Eason et al., 1965; Geldreich, 1939; Hajcak et al., 2004; Jacobs et al., 1994), but also performance on Go/Nogo tasks (Kato et al., 2009; Kato et al., 2012; Scholz et al., 2009; Schulz et al., 2007; Smallwood et al., 2009). From this perspective, it may be useful in future studies to include additional state measures to further clarify the effect of these energetic factors during cognitive training.

\subsection{Early ERP components}

Interestingly, training differentially modulated Go/Nogo N1 amplitudes between conditions. Participants in the Low and Medium conditions showed relatively similar N1 amplitudes to Go and Nogo stimuli by the end of training, while those in the High condition displayed a reduced Nogo relative to Go N1. The N1 ERP component is sensitive to the sensory attributes of stimuli and is modulated by attention (Näätänen and Picton, 1987). Similarly, Bekker et al. (2005) has also suggested that N1 amplitudes may index an attentional switch that is determinative for subsequent inhibitory control. Together with the performance data, these findings point to differential focusing on task requirements for each of the three difficulty conditions over the course of the training session. That is, in the context of improved Go/Nogo proficiency, it would seem that participants in both the Low and Medium conditions applied a more balanced approach in attending to Go and Nogo stimuli. By contrast, training seems to have led to a reduction in attentional resources being directed towards Nogo stimuli in the High condition, possibly due to these participants focusing primarily on the Go stimulus in order to respond within the strict RTD (Johnstone et al., 2005). Such a reduction in attention to the Nogo stimuli may, in part, explain the increased errors on Nogo stimuli with training in this group.

Notably, training resulted in different changes in P2 topography between the task difficulty conditions: the Medium and High condition showed a more anterior P2 to both Go and Nogo stimuli, contrasting with the Low condition which displayed an enhanced centroparietal Nogo compared to the Go P2 (see Figure $4 \mathrm{c}$ and d). These differences were apparent in the vectorscaled data suggesting a training-induced shift in the neural generators of these components (Johnson, 1993). Moreover, although the P2 component is generally thought to index the appropriate classification of stimuli (Oades, 1998), its functional significance may have dissociable meanings according to scalp location. A more anterior P2 may index greater 
relevance of task stimuli (Potts, 2004; Potts et al., 1996), whereas larger parietal P2s have been reported during easy perceptual learning paradigms, paralleled by improvements in performance (Ding et al., 2003; Qu et al., 2010; Song et al., 2002; Song et al., 2005). Thus, a more anterior Go and Nogo P2 for the Medium and High conditions implies greater stimulus processing and evaluation of these stimuli with training, possibly due to the faster overall Go RT for these conditions. By contrast, the increased Nogo P2 over centroparietal sites for the Low condition may index an early perceptual learning effect that is linked to more automated bottom-up processing in a relatively easy task, as implied by inhibition performance at ceiling for this condition.

Consistent with this notion, Verbruggen and Logan (2008) posit that Go/Nogo task practice leads to the emergence of automatic inhibition, where learned associations between stimuli and withholding a response reduce the need for top-down executive control. In this context, it may be that enhanced Nogo P2 amplitudes for well-learned or easy tasks reflects the automated inhibition of Nogo stimuli, freeing top-down mechanisms from further processing. In contrast, more difficult tasks appear to show a reduced P2 at parietal sites, potentially signalling the need for greater top-down inhibitory control at later processing stages (Dimoska and Johnstone, 2008; Smith et al., 2004).

\subsection{Inhibition-related ERP components}

N2 amplitude and latency decreased across the session regardless of condition, in line with previous research (Ding et al., 2003; Song et al., 2002). Interestingly, however, further interactions highlighted that this decline was primarily due to reduction of the Go, but not the Nogo N2. The N2 component is thought to represent a controlled mismatch detection process (Näätänen and Picton, 1986; Snyder and Hillyard, 1976), and is therefore related to stimulus discrimination (Johnstone et al., 2001; Johnstone et al., 1996; Ritter et al., 1983). Attenuated cortical responses to repeated stimuli have typically been interpreted as an early manifestation of learning-induced neural plasticity (see Garrido et al., 2008; Race et al., 2010; Summerfield et al., 2011). Thus, reduced Go N2 amplitudes across training blocks may suggest more efficient stimulus discrimination with training. Moreover, Neubauer and Fisk (2009) have proposed that greater neural efficiency might arise when training with tasks of low difficulty. If this is the case, the finding of the larger training-induced decline in N2 latency for the Low than Medium/High conditions is in accord with this interpretation.

Against predictions, training-related variations in task performance were not accompanied by an increase in the Nogo N2 (see Figure 4). While consistent with some previous reports 
investigating the effect of repetition (Falkenstein et al., 2002; Kato et al., 2009), it is in contrast to others suggesting enhancements in this component concurrent with improvements in Go/Nogo proficiency (for a discussion see Manuel et al., 2010; Schapkin et al., 2007). Our previous work, which considered the effect of decreasing RTDs (Benikos et al., in press), found that faster Go RTs resulted in incremental increases in Nogo N2 amplitude, interpreted in terms of enhanced response conflict (see also Nieuwenhuis et al., 2003; Smith, 2011). From this perspective, it may be argued that the stable Nogo N2 in the present study reflects a reduction in the relative level of response conflict, given that all three conditions showed declines in Go RT. Nonetheless, it is interesting to note that the only inhibition training study which reported changes in Nogo N2 amplitude, found this effect after three days of training (Schapkin et al., 2007), while Luu and colleagues (2007) have suggested that changes in the N2 may only be apparent during the later stages of learning. Thus, combined with the fact that participants were presented with fewer Nogo than Go stimuli over the course of the training session (i.e. $30 \%$ vs. $70 \%$ ), the equivalent Nogo N2 may be due to the slower time course of learning for this component.

While little change was seen across the session for the Low condition, the Nogo > Go P3 effect increased as a function of task difficulty particularly over central regions; showing a greater training-induced augmentation for the High than Medium condition (Figure 5). Since previous research has reported no significant change (e.g. Jodo and Inoue, 1990; Johnstone et al., 2010; Schapkin et al., 2007), this result is the first to suggest that increased task difficulty may be required to elicit training-related enhancements in the Nogo P3. Does this effect represent a strengthening of a top-down inhibition mechanism? We have previously shown that increased inhibition difficulty results in reduced Nogo P3 amplitudes (Benikos et al., in press). An implication here is that P3 amplitude is inversely related to inhibitory load. On this basis, it could be suggested that conditions like practice which tend to reduce task difficulty result in increased Nogo P3 amplitudes (for a similar interpretation regarding memory load see Kok, 1997). Similarly, the training-induced increase in Nogo P3 for the Medium condition accompanied by enhanced Go/Nogo proficiency may be interpreted in this manner. Moreover, despite increased Nogo errors, the greatest Nogo P3 for the High condition may not completely rule-out a practice-based interpretation of this effect; given that Nogo errors plateaued by block 4 for this condition, while the Nogo P3 continued to increase until the conclusion of training. This suggests a continued adaption to the difficulty of the task and is perhaps unsurprising, considering the brief (42 min) experience participants had with the training and its high difficulty level (for a similar fMRI finding see Kelly et al., 2006b). While it may also be argued that a larger Nogo P3 over central regions could simply be due to greater monitoring of the 
inhibition outcome in order to limit the error rate (for a similar argument see Beste et al., 2010), this explanation is unlikely, given the location of this component over the pre-motor cortex and that central increases in the Nogo P3 have been suggested to reflect a motoric inhibition process unrelated to movement related potentials (Smith et al., 2007). Since previous training studies have reported that neural changes can precede behavioural changes (Atienza et al., 2002), and that training in higher-order executive functions can potentially transfer to untrained tasks (Dahlin et al., 2008), an avenue for future research would be to investigate whether traininginduced enhancements in Nogo P3 transfers to unpractised Go/Nogo stimuli. If larger Nogo P3s represent an enhancement in inhibitory control processes, this improvement would be expected to transfer to the untrained stimuli.

Finally, future studies could consider the influence of differences in IQ and potential learning capacity between training conditions. While previous research has generally reported no relationship between IQ and baseline inhibitory performance (Friedman et al., 2006), IQ is a potentially strong predictor of learning ability (Alloway and Alloway, 2010). Thus, it may be helpful for future research to include an index of IQ and potentially other individual differences which potentially interact with training-related gains in inhibitory performance (e.g. impulsivity; Horn et al., 2003).

\subsection{Conclusions}

In summary, this study provides novel evidence for the differential effects of task difficulty on the training of inhibitory control. In particular, the behavioural effects of short-term training appear to be optimised during conditions of moderate rather than low or high inhibitory load. An across-session increase in task-related arousal did not differ between conditions, indicating a generalised increase in demand for mental resources with time-on-task. Moreover, taken together the findings of the present study are of relevance to the theoretical accounts of the effect of training on inhibition-related neural activity. While changes associated with training have typically either been linked to the reinforcement of top-down executive control processes, or to the emergence of automatic bottom-up forms of inhibitory control, our results imply that these effects may be dependent on task demands. Whereas conditions of Low task difficulty may primarily lead to early bottom-up perceptual learning as reflected by enhancements in the centroparietal Nogo P2, top-down changes, particularly in the Nogo P3 appear to be associated with enhanced task difficulty. Although further research is needed in this area, these findings implicate the potentially key role of task difficulty for researchers attempting to design effective inhibition training paradigms to ameliorate inhibitory control deficits as seen in disorders such as ADHD. 


\section{References}

Ahissar, M., Hochstein, S., 2004. The reverse hierarchy theory of visual perceptual learning. Trends in Cognitive Sciences 8, $457-464$.

Alloway, T.P., Alloway, R.G., 2010. Investigating the predictive roles of working memory and IQ in academic attainment. Journal of Experimental Child Psychology 106, 20-29.

Aron, A.R., Durston, S., Eagle, D.M., Logan, G.D., Stinear, C.M., Stuphorn, V., 2007. Converging Evidence for a Fronto-Basal-Ganglia Network for Inhibitory Control of Action and Cognition. The Journal of Neuroscience 27, 1186011864.

Aron, A.R., Robbins, T.W., Poldrack, R.A., 2004. Inhibition and the right inferior frontal cortex. Trends in Cognitive Sciences 8170 - 177.

Atienza, M., Cantero, J.L., Dominguez-Marin, E., 2002. The Time Course of Neural Changes Underlying Auditory Perceptual Learning. Learning and Memory 9, 138-150.

Band, G.P.H., Ridderinkhof, K.R., van der Molen, M.W., 2003. Speed accuracy modulation in case of conflict: the roles of activation and inhibition. Psychological Research 67, $266-279$.

Barry, R.J., Sokolov, E.N., 1993. Habituation of phasic and tonic components of the orienting reflex. Journal of Psychophysiology 15, 39-42.

Bekker, E.M., Kenemans, J.L., Hoeksm, M.R., Talsm, D., Verbaten, M.N., 2005. The Pure Electrophysiology of Stopping. . International Journal of Psychophysiology 55, $191-198$.

Benikos, N., Johnstone, S.J., Roodenrys, S., in press. Varying task difficulty in the Go/Nogo task: the roles of inhibition, arousal and effort. International Journal of Psychophysiology. 
Beste, C., Saft, C., Andrich, J., Gold, R., Falkenstein, M., 2008. Response inhibition in Huntington's disease - a study using ERPs and sLORETA. Neuropsychologia 46, 1290 - 1297.

Beste, C., Willemssen, R., Saft, C., Falkenstein, M., 2010. Response inhibition subprocesses and dopaminergic pathways: Basal ganglia disease effects. Neuropsychologi 48, 366-373.

Blumenthal, D.M., Gold, M.S., 2012. Neurobiology of food addiction. Current Opinion in Clinical Nutrition and Metabolic Care 13, 359 - 365.

Bradley, J.V., 1958. Complete counterbalancing of immediate sequential effects in a Latin square design. Journal of the American Statistical Association. 53, 525-528.

Bruin, K.J., Wijers, A.A., van Staveren, A.S., 2001. Response priming in a Go/Nogo task: Do we have to explain the Go/Nogo N2 effects in terms of response activation instead of inhibition? Clinical Neurophysiology 112, 660 1671.

Buonomano, D.V., Merzenich, M.M., 1998. Cortical plasticity: from synapses to maps. Annual Review of Neuroscience 21, 149-186.

Chein, J.M., Morrison, A.B., 2010. Expanding the mind's workspace: training and transfer effects with a complex working memory span task. Psychonomic Bulletin \& Review 17, 193-199.

Cooper, R., Shallice, T., 2000. Contention sceduling and control of routine activities. Cognitive Neuropsychology 17, 297.

Cramer, S.C., Sur, M., Dobkin, B.H., O'Brien, C., Sanger, T.D., Trojanowski, J.Q., Rumsey, J.M., Hicks, R., Cameron, J., Chen, D., Chen, W.G., Cohen, L.G., Decharms, C., Duffy, C.J., Eden, G.F., Fetz, E.E., Filart, R., Freund, M., Grant, S.J., Haber, S., Kalivas, P.W., Kolb, B., Kramer, A.F., Lynch, M., Mayberg, H.S., McQuillen, P.S., Nitkin, R., Pascual-Leone, A., Reuter-Lorenz, 
P., Schiff, N., Sharma, A., Shekim, L., Stryker, M., Sullivan, E.V., Vinogradov, S.t., 2011. Harnessing neuroplasticity for clinical applications. Brain 134, 1591-1609.

Dagenbach, D., Carr, T.H., 1994. Inhibitory processes in attention, memory and language. Academic Press, San Diego, CA.

Dahlin, E., Nyberg, L., Backman, L., Neely, A.S., 2008. Plasticity of executive functioning in young and older adults: Immediate training gains, transfer, and long-term maintenance. Psychology and Aging 23, 720 - 730.

Dawson, M.E., Schell, A.M., Filion, D.L., 1990. The electrodermal system, in: Cacioppo, J.T., Tassinary, L.G. (Eds.), Principles of psychophysiology: Physical, social, and inferential elements. Cambridge University Press, New York, pp. 295-324.

Dempster, F.N., Corkill, A.J., 1999. Interference and inhibition in cognition and behavior: Unifying themes for educational psychopathology. Psychology Review 11, 1-88.

Dimoska, A., Johnstone, S.J., 2008. Effect of varying stop-signal probability on ERPs in the stop signal task: Do they reflect variations in inhihibitory processing or simply novelty effects? Biological Psychology 77, 324-336. Ding, Y., Song, Y., Fan, S., Qu, Z., Chen, L., 2003. Specificity and generalization of visual perceptual learning in humans: an event-related potential study. Neuroreport 14, 587-590.

Dowsett, S.M., Livesey, D.J., 2000. The development of inhibitory control in preschool children: effects of "executive skills" training. Developmental Psychobiology 362, $161-174$.

Eason, R.G., Beardshall, A., Jaffee, S., 1965. Performance and physiological indicants of activation in a vigilance task. Perceptual and Motor Skills 20, 313. 
Falkenstein, M., Hoormann, J., Christ, S., Hohnsbein, J., 2000. ERP components on reaction errors and their functional significance: a tutorial. Biological Psychology 51, 87 - 107.

Falkenstein, M., Hoormann, J., Hohnsbein, J., 1999. ERP components in Go/Nogo tasks and their relation to inhibition. Acta Psychologica 101, 267 291.

Falkenstein, M., Hoormann, J., Hohnsbein, J., 2002. Inhibition-related ERP components: Variation with modality, age and time-on-task. Journal of Psychophysiology 16, 167-175.

Friedman, R., Miyake, A., Corley, R.P., Young, S.E., Defries, J.C., Hewitt, J.K., 2006. Not All Executive Functions Are Related to Intelligence. Psychological Science 17, 172-179.

Garrido, M.I., Friston, K.J., Kiebel, S.J., Stephan, K.E., Baldeweg, T., Kilner, J.M., 2008. The functional anatomy of the MMN: a DCM study of the roving paradigm. Neuroimage 42, $936-944$.

Geldreich, E.W., 1939. Skin Conductance Changes Occurring during Mental Fatigue. Transactions of the Kansas Academy of Science 42, 393-395.

Green, C.S., Bavelier, D., 2008. Exercising your brain: A review of human brain plasticity and training-induced learning. Psychology of Aging 23, 692701.

Hajcak, G., McDonald, N., Simons, R.F., 2004. Error-related psychophysiology and negative affect. Brain and Cognition 56, 189 - 197.

Hester, R., Murphy, K., Garavan, H., 2004. Beyond commond resources: The cortical bassis for resolving task interference. NeuroImage 23, 202-213. Horn, N.R., Dolan, M., Elliott, R., Deakin, J.F., Woodruff, P.W., 2003. Response inhibition and impulsivity: an fMRI study. Neuropsychologia 41, 1959-1966. 
Houben, K., 2011. Overcoming the urge to splurge: Influencing eating behavior by manipulating inhibitory control. Journal of Behavior Therapy and Experimental Psychiatry 42, 384-388.

Houben, K., Jansen, A., 2011. Training inhibitory control: A recipe for sweet temptations. Appetite 56, 345-349.

Houben, K., Nederkoorn, C., Wiers, R.W., Jansen, A., 2011. Resisting temptation: decreasing alcohol-related affect and drinking behavior by training response inhibition. Drug and Alcohol Dependence 16, 132-136.

Howell, D.C., 2009. Statistical Methods for Psychology, 7th ed. Wadsworth Belmont, CA.

Jacobs, S.C., Friedman, R., Parker, J.D., Tofler, G., H., Jimenez, A.H., Muller, J.E., Benson, H., Stone, P.H., 1994. Use of skin conductance changes during mental stress testing as an index of autonomic arousal in cardiovascular research. American Heart Journal 28, 1170-1177.

Jasper, H.H., 1958. Report of the committee on methods of clinical examination in electroencephalography. Electroencephalography and Clinical Neurophysiology 10, 370-375.

Jodo, E., Inoue, K., 1990. Effects of practice on the P300 in a Go/NoGo task. EEG Clinica Neurophysiology 76, 249 - 257.

Jodo, E., Kayama, Y., 1992. Relation of a negative ERP component to response inhibition in a Go/No-go task. . Electroencephalography Clinical Neurophysiology 82, $477-482$.

Johnson, J., R. , 1993. On the neural generators of the P300 component of the event-related potential. Psychophysiology 30, 90-97. Johnstone, S.J., Barry, R.J., Anderson, J.W., 2001. Topographic distribution and developmental timecourse of auditory event-related potentials in two 
subtypes of attention-deficit hyperactivity disorder. International Journal of Psychophysiology 42, 73-94.

Johnstone, S.J., Barry, R.J., Anderson, J.W., Coyle, S.F., 1996. Age-related changes in child and adolescent event-related potential component morphology, amplitude and latency to standard and target stimuli in an auditory oddball task. International Journal of Psychophysiology 24, 223-238. Johnstone, S.J., Pleffer, C.B., Barry, R.J., Clarke, A.R., Smith, J.L., 2005. Development of inhibitory processing during the Go/Nogo task. Journal of Psychophysiology 19, 11-23.

Johnstone, S.J., Roodenrys, S., Phillips, E., Watt, A.J., Mantz, S., 2010. Combined working memory and inhibition training for children with AD/HD. ADHD Attention Deficit and Hyperactivity Disorders 2, 31-42. Johnstone, S.J., Watt, A.J., Dimoska, A., 2009. Varying required effort during intereference control in children with AD/HD: Task performance and ERPs. International Journal of Psychophysiology 76, 174-185.

Kato, Y., Endo, H., Kizuka, T., 2009. Mental fatigue and impaired response processes: Event-related brain potentials in a Go/Nogo task. International Journal of Psychophysiology 72, 204-211.

Kato, Y., Endo, H., Kobayakawa, T., Kato, K., Kitazaki, S., 2012. Effects of intermittent odours on cognitive-motor performance and brain functioning during mental fatigue. Ergonomics 1.

Kelley, T.A., Yantis, S., 2009. Learning to attend: Effects of practice on information selection. Journal of Vision 9, 1 - 18.

Kelly, C., Foxe, J.J., Garavan, H., 2006a. Patterns of normal human brain plasticity after practice and their implications for neurorehabilitation. Archives of Physical Medicine and Rehabilitation 87, S20-S29.

Kelly, C., Garavan, H., 2005. Human Functional Neuroimaging of Brain Changes Associated with Practice. Cerebral Cortex 15, 1089-1102. 
Kelly, C., Hester, R., Foxe, J.J., Shpaner, M., Garavan, H., 2006b. Flexible cognitive control: Effects of individual differences and brief practice on a complex cognitive task. NeuroImage 31, 866-886.

Klingberg, T., 2010. Training and plasticity of working memory. Trends in Cognitive Sciences 14, 317-324.

Klingberg, T., Fernell, E., Olesen, P.J., Johnson, M., Gustafsson, P., Dahlström, K., Gillberg, C.G., Forssberg, H., Westerberg, H., 2005. Computerized training of working memory in children with ADHD - a randomized, controlled trial. Journal of the American Academy of Child and Adolescent Psychiatry 44, 177-186.

Klingberg, T., Forssberg, H., Westerberg, H., 2002. Training of working memory in children with ADHD. Journal of Clinical and Experimental Neuropsychology 24, $781-791$.

Kok, A., 1986. Effects of degradation of visual stimuli on components of the event-related potential (ERP) in go/nogo reaction tasks. Biological Psychology 23, 21-38.

Kok, A., 1997. Event-related-potential (ERP) reflections of mental resourc s: a review and synthesis. Biological Psychology 45, 19-56.

Kujal, T., Näätänen, R., 2010. The adaptive brain: a neurophysiological perspective. Progress in Neurobiology 91, 55-67.

Larue, G.S., Rakotonirainy, A., Pettitt, A.N., 2011. Driving performance impairments due to hypovigilance on monotonous roads. Accident Analysis \& Prevention 43, 2037-2046.

Lillard, A., Erisar, A., 2011. Old dogs learning new tricks: Neuroplasticity beyond the juvenile period. Developmental Review 31, 207-239.

Lindqvist, S., Thorell, L.B., 2009. A brief report: Manipulating task difficulty in laboratory measures of inhibitory control. Child Neuropsychology 15, 1-7. 
Luu, P., Tucker, D.M., Stripling, R., 2007. Neural mechanisms for learning actions in context. . Brain Research 1179, 89 - 105.

Manuel, A.L., Grivel, J., Bernasconi, F., Murray, M.M., Spierer, L., 2010. Brain Dynamics Underlying Training-Induced Improvement in Suppressing Inappropriate Action. Journal of Neuroscience 30, 13670-13678.

McCarthy, G., Wood, C.C., 1985. Scalp distributions of event-related potentials: an ambiguity associated with analysis of variance models. Electroencephalography and Clinical Neurophysiology 62, 203 - 208. McNab, F., Varrone, A., Farde, L., Jucaite, A., Bystritsky, P., Forssberg, H., Klingberg, T., 2009. Changes in cortical dopamine D1 receptor binding associated with cognitive training. Science 323, $800-802$.

Monsell, S., 2003. Task switching. Trends in Cognitive Science 7, 134-140. Näätänen, R., Picton, T., 1986. N2 and automatic versus controlled processes, in: McCallum, W.C., Zappoli, R., Denoth, F. (Eds.), Cerebral Psychophysiology. Elsevier, Amsterdam, pp. 171-188.

Näätänen, R., Picton, T., 1987. The N1 wave of the human electric and magnetic response to sound: a review and an analysis of the component structure. . Psychophysiology $24375-425$.

Naccache, L., Dehaene, S., Cohen, L., Habert, M., Guichart-Gomez, E., Galanaude, D., Willera, J., 2005. Effortless control: executive attention and conscious feeling of mental effort are dissociable. Neuropsychologia 43, 13181328.

Neubauer, A.C., Fink, A., 2009. Intelligence and neural effiiciency. Neuroscienece and Biobehavioral Reviews 33, 1004-1023. Nieuwenhuis, S., Yeung, N., van den Wildenburg, W., Ridderinkhof, K.R., 2003. Electrophysiological correlates of anterior cingulate function in a 
go/nogo task: effects of response conflict and trial type frequency. Cognitive and Affective Behavioral Neuroscience 3, $17-26$.

Olesen, P.J., Westerberg, H., Klingberg, T., 2004. Increased prefrontal and parietal activity after training of working memory. Nature Neuroscience 7, 7579.

Picton, T.W., Bentin, S., Berg, P., Donchin, E., Hillyard, S.A., Johnson, R.J., Miller, G.A., Ritter, W., Ruchkin, D.S., Rugg, M.D., Taylor, M.J., 2000.

Guidelines for using human event-related potentials to study cognition: Recording standards and publication criteria. Psychophysiology 37, 127-152. Potts, G.F., 2004. An ERP index of task relevance evaluation of visual stimuli. Brain and Cognition 56, 5 - 13.

Potts, G.F., Liotti, M., Tucker, D.M., Posner, M.I., 1996. Frontal and inferior temporal cortical activity in visual target detection: evidence from high spatially sampled event-related potentials. Brain Topography 9, 3 - 14 . Qu, Z., Song, Y., Ding, Y., 2010. ERP evidence for distinct mechanisms of fast and slow visual perceptual learning. Neuropsychologia 48, 1869-1874. Race, E.A., Badre, D., Wagner, A.D., 2010. Multiple forms of learning yield temporally distinct electrophysiological repetition effects. Cerebral Cortex 20, $1726-1738$.

Randall, W.M., Smith, J.L., 2011. Conflict and inhibition in the cued-Go/Nogo task. Clinical Neurophysiology 122, 2400-2407.

Raskin, S., Mills, G.N., Garbino, J.T., 2011. Practice-related changes in brain activity, in: Raskin, S. (Ed.), Neuroplasticity and Neurorehabilitation. . Guilford, London, pp. 103-114.

Ravden, D., Polich, J., 1998. Habituation of P300 from visual stimuli. International Journal of Psychophysiology 30, 359-365. 
Ritter, W., Simson, R., Vaughan, H.G., 1983. Event-related potential correlates of two stages of information processing in physical and semantic discrmination tasks. Psychophysiology 20, 168-179.

Roche, R.A.P., Garavan, H., Foxe, J.J., O’ Mara, S.M., 2005. Individual differences discriminate event-related potentials but not performance during response inhibition. Experimental Brain Research 160, 60-70.

Rueda, M.R., Rothbart, M.K., McCandliss, B.D., Saccomanno, L., Posner, M.I., 2005. Training, maturation, and genetic influences on the development of executive attention. Proceedings of the National Academy of Sciences 102 $14931-14936$.

Schapkin, S.A., Falkenstein, M., Marks, A., Griefahn, B., 2007. Practicerelated effects in a Go-Nogo task. Perceptual and Motor Skills 105, 1275-1288. Scholz, U., La Marca, R., Nater, U.M., Aberle, I., Ehlert, U., Hornung, R., Martin, M., Kliegel, M., 2009. Go no-go performance under psychosocial stress: Beneficial effects of implementation intentions. Neurobiology of Learning and Memory 91, $89-92$.

Schulz, K.P., Fan, J., Magidina, O., Marks, D.J., Hahn, B., Halperi, n.J.M., 2007. Does the Emotional Go/No-Go Task Really Measure Behavioral Inhibition? Convergence with Measures on a Non-Emotional Analog. Archives of Clinical Neuropsychology 22, 151-160.

Semlitsch, H.V., Anderer, P., Schuster, P., Presslich, O.A., 1986. A solution for reliable and valid reduction of ocular artifacts, applied to the P300 ERP. Psychophysiology 23, $695-703$.

Slagter, H.A., Davidson, R.J., Lutz, A., 2011. Mental training as a tool in the neuroscientific study of brain and cognitive plasticity. Frontiers in Human Neuroscience 5, 1-12. 
Smallwood, J., Fitzgerald, A., Miles, L.K., Phillips, L.H., 2009. Shifting Moods, Wandering Minds: Negative Moods Lead the Mind to Wander. Emotion 9, 271276.

Smith, J.L., 2011. To Go or not to Go, that is the question: Do the N2 and P3 reflect stimulus- or response-related conflict? International Journal of Psychophysiology 82, 143-152.

Smith, J.L., Douglas, K.M., 2011. On the use of event-related potentials to auditory stimuli in the Go/NoGo task. Psychiatry Research - Neuroimaging $193,177-181$.

Smith, J.L., Johnstone, S.J., Barry, R.J., 2004. Inhibitory processing during the Go/Nogo task: an ERP analysis of children with attentiondeficit/hyperactivity disorder. Clinical Neurophysiology 115, 1320 - 1331. Smith, J.L., Johnstone, S.J., Barry, R.J., 2006. Effects of pre-stimulus processing on subsequent events in a warned Go/NoGo paradigm: Response preparation, execution, and inhbition. International Journal of Psychophysiology 61, 121-133.

Smith, J.L., Johnstone, S.J., Barry, R.J., 2007. Response priming in the Go/NoGo task: the N2 reflects neither inhibition nor conflict. . Clinical Neurophysiology $118,343-355$.

Smith, J.L., Johnstone, S.J., Barry, R.J., 2008. Movement-related potentials in the Go/NoGo task: the P3 reflects both cognitive and motor inhibition. Clinical Neurophysiology 119, $704-714$.

Smith, J.L., Smith, E., Provost, A.L., Heathcote, A., 2010. Sequence effects support the conflict theory of N2 and P3 in the Go/NoGo task. International Journal of Psychophysiology 75, 217-226.

Snyder, E., Hillyard, S.A., 1976. Long-latency potentials to irrelevant, deviant stimuli. Behavioral Biology 16, 319-331. 
Song, Y., Ding, Y., Fan, S., Chen, D., 2002. An event-related potential study of visual perceptual learning under short-term and long-term training conditions. Neuroreport 13, 2053-2057.

Song, Y., Ding, Y., Fan, S., Qu, Z., Xu, L., Lu, C., Peng, D., 2005. Neural substrates of visual perceptual learning of simple and complex stimuli. Clinical Neurophysiology 116, $632-639$.

Spencer, K.M., Dien, J., Donchin, E., 2001. Spatiotemporal analysis of the late ERP responses to deviant stimuli. Psychophysiology 38, 343-358.

Summerfield, C., Wyart, V., Johnen, V.M., de Gardelle, V., 2011. Human scalp electroencephalography reveals that repetition suppression varies with expectation. Frontiers in Human Neuroscience 5, 67.

Tabachnick, B.G., Fidell, L.S., 1996. Using Multivariate Statistics. HarperCollins, New York.

Tang, Y.Y., Posner, M.I., 2009. Response to Jaeggi et al.: Exploring training methods. Trends in Cognitive Neuroscience 13, 192-193.

Thomas, S.J., Gonsalvez, C.J., Johnstone, S.J., 2009. Sequence effects in the Go/NoGo task: Inhibition and facilitation. International Journal of Psychophysiology 74, 209-219.

Thorell, L.B., Lindqvist, S., Bergman-Nutley, S., Bohlin, G., Klingberg, T., 2009. Training and transfer effects of executive functions in preschool children. Developmental Science 12, $106-113$.

Tomporowski, P.D., 2003. Performance and perceptions of workload amoung young and older adults: Effects of practice during cognitively demanding tasks. Educational Gerontlogy 29, 447-466.

Veling, H., Aarts, H., Papies, E.K., 2011. Using stop signals to inhibit chronic dieters' responses toward palatable foods. Behavior Research Therapy 49, 771-780. 
Verbruggen, F., Logan, G.D., 2008. Automatic and controlled response inhibition: Associative learning in the Go/Nogo and Stop-Signal paradigms. Journal of Experimental Psychology: General 137, 619-672.

Wang, Y., Song, Y., Qu, Z., Ding, Y., 2010. Task difficulty modulates electrophysiological correlates of perceptual learning. International Journal of Psychophysiology $75234-240$.

Wiers, R.W., Bartholow, B.D., van den Wildenberg, E., Thush, C., Engels, R.C., Sher, K.J., Grenard, J., Ames, S.L., Stacy, A.W., 2007. Automatic and controlled processes and the development of addictive behaviors in adolescents: a review and a model. Pharmacology Biochemistry and Behavior 86, 263-283.

Yerkes, R.M., Dodson, J.D., 1908. The relation of strength of stimulus to rapidity of habit-formation. Journal of Comparative Neurology 18, $459-482$. 